\title{
Defect Dynamics for Spiral Chaos in Rayleigh-Bénard Convection
}

\author{
M. C. Cross and Yuhai Tu* \\ Condensed Matter Physics, Caltech, Pasadena, California 91125
}

(Received 5 October 1994)

\begin{abstract}
A theory of the novel spiral chaos state recently observed in Rayleigh-Bénard convection is proposed in terms of the importance of invasive defects, i.e., defects that through their intrinsic dynamics expand to take over the system. The motion of well developed spiral defects is dominated by wave vector frustration, rather than a rotational motion driven by a vertical vorticity field. This leads to a continuum of spiral frequencies, and a spiral may rotate in either sense depending on the wave vector of its local environment. Results of extensive numerical work on equations modeling the convection system provide confirmation of these ideas.
\end{abstract}

PACS numbers: 47.20.Bp, 47.27.Te

A novel spatiotemporal chaotic state in RayleighBénard convection consisting of dynamic spirals or targets has recently been discovered experimentally [1,2], and then reproduced numerically first in equations that approximately model the system [3] and then in the full thermally driven Navier-Stokes equations [4]. This "spiral chaos" state is particularly interesting because it occurs for parameter values where the familiar state of straight parallel convecting rolls (the "stripe" state) is known to be stable, and because the spiral geometry, although familiar in chemical reaction diffusion systems, was completely unexpected in this system where the ideal state is static. The spiral state also provides an intriguing example of the phenomenon of "defect chaos" where persistent dynamics is apparently associated with easily identified defects or coherent structures. Although numerical work has reproduced the experimental results with remarkable accuracy, there is still little theoretical understanding why the spiral chaotic state develops, or of the dynamic behavior of the spiral or target defects within the chaotic state.

In this paper we introduce the idea of an invasive defect-a localized structure that through its intrinsic dynamics tends to grow and take over a significant portion of the system - as a way to understand the instability (or rather, lack of persistence) of nonideal states towards the chaotic state. We analyze the dynamics of individual spirals and targets based on a slow-variation approach known as "phase equations" and show that "wave vector frustration" is crucial to understand this dynamics. Perhaps surprisingly, fluid vorticity plays a secondary role in the dynamics of the spirals: The spirals appear to rotate because of the radial motion of the rolls induced by wave vector frustration exactly as in the radial motion of the axisymmetric target states. This leads us to a prediction for when the spiral chaos state should be expected. We analyze the transition between a spiral dominated state and a target dominated state in terms of a novel core instability of an axisymmetric target to nonaxisymmetric perturbations. In addition, we present results of extensive numerical investigations of classes of model equations that allow us to test these ideas, and for the first time numerically demonstrate the spiral to target transition.

Our theoretical analysis is based on the "phase equation" [5] - a dynamical equation for a variable describing displacements of a local stripe pattern. An important feature of the convection system is that this dynamics is coupled to an additional long wavelength mode consisting of a horizontal fluid flow with nonzero mean averaged over the depth of the cell. Such a flow is induced by distortions of the convective rolls (e.g., curvature) and in turn advects the rolls. Since the mean flow must be divergence free by the near incompressibility of the fluid, it takes the form of a stirring motion, and may be completely described in terms of a vertical vorticity $\Omega$. Previous work $[3,4]$ on the spiral chaos has suggested that the vertical vorticity resulting from the chiral nature of the spiral state may be important in the observed dynamics of the spirals. We find that the role of $\Omega$ is more subtle than this.

The phase variable $\theta(\vec{x}, t)$ is defined as a function of time and the horizontal coordinate $\vec{x}=(x, y)$ so that the local wave vector is $\vec{q}=\vec{\nabla} \theta$. An expansion in slow variations of a basic stripe pattern yields the equation [5]

$$
\dot{\theta}+\vec{U} \cdot \vec{q}=\tau^{-1}(q) \vec{\nabla} \cdot[\vec{q} B(q)],
$$

where $\vec{U}$ is the divergence free mean flow completely characterized by the vertical vorticity $\Omega=\hat{z} \cdot \vec{\nabla} \times \vec{U}$, in turn driven by distortions of the stripes

$$
\Omega=-\gamma(q) \hat{z} \cdot \vec{\nabla} \times\left\{\vec{q} \vec{\nabla} \cdot\left[\vec{q} A^{2}(q)\right]\right\} .
$$

In these expressions $\tau, \gamma, A$, and $B$ are functions of the magnitude of the local wave vector and depend on the control parameter as well as other fluid parameters. In (2) we have used the simplified form valid near threshold [5]: The complicated expression valid more generally $[6,7]$ should not change the conclusions.

From these equations we show that the spiral dynamics is induced by the radial motion of rolls forced by wave vector frustration rather than by a rotation of the structure driven by a vorticity field induced by the chiral structure. Since the wave vector frustration depends on the environment of each spiral, the rotation frequencies 
may take on a continuum of values instead of a unique value as found for chemical spirals [7]. In addition, a spiral of a given chiral sense may rotate in either direction depending on its environment. Note, however, that spirals that rotate against their chirality will unwind and disappear from the system, so that a prevalence of corotating spirals is expected.

These results are readily obtained by integrating (1) for the phase of a spiral $\theta=k(r) r+m \phi-\omega t$ over a cylindrical geometry radius $R$ with $R \gg 1$ for a well developed spiral:

$$
\begin{aligned}
\omega \int d^{2} r \tau(q)= & \int d^{2} r \tau(q) \vec{U} \cdot \vec{q} \\
& -\left.\int B(q) q_{r} r d \phi\right|_{r=R} .
\end{aligned}
$$

(Here $r$ and $\phi$ are cylindrical polar coordinates.) The second term on the right, which is also present in the axisymmetric target case, is zero if $q=q_{f}$, the "focus selected wave number" [8] defined by $B\left(q_{f}\right)=0$ [5], and is nonzero only if the wave number is held away from this value by some other means (wave vector frustration). In this case the contribution to $\omega$ is of order $\tau^{-1} B^{\prime}\left(q_{f}\right) q_{f}\left(q-q_{f}\right) R^{-1}$ with $\tau$ an average of $\tau(q)$ over the cell. We can estimate the size of the first term by correspondingly integrating the vorticity equation (2)

$$
\begin{aligned}
\int d^{2} r[\gamma(q)]^{-1} \Omega= & -\oint_{R} \vec{\nabla} \cdot\left(\vec{q} A^{2}\right) \vec{q} \cdot d \vec{l} \\
& +\oint_{r \rightarrow 0} \vec{\nabla} \cdot\left(\vec{q} A^{2}\right) \vec{q} \cdot d \vec{l} .
\end{aligned}
$$

It is readily checked that for $R \gg 1$ where $A^{2} \rightarrow$ const the first term on the right-hand side goes as $R^{-1}$. If the amplitude $A$ remained constant as $r \rightarrow 0$, the second term would diverge as $r^{-1}$. Instead $A \rightarrow 0$ over the coherence length $\xi$ for spatial variations of the amplitude of the pattern, to give a large but finite contribution. Thus the integrated vorticity is dominated by a contribution localized in the core region where amplitude and phase are rapidly varying, consistent with numerics [3]. Now $\vec{U} \cdot \vec{q} \simeq$ $U_{\phi} m / r \sim m \bar{\Omega} \xi^{2} / r^{2}$ with $\bar{\Omega}$ an average vorticity, so that the first term in (3) gives a contribution to the frequency $\omega$ proportional to $R^{-2}$, smaller by the factor $R^{-1}$.

The invasive nature of spirals and targets rests on the comparison of the background wave number $q_{b}$ with $q_{f}$ : If $q_{b}>q_{f}$ then rolls will tend to move into the defect center, a spiral will "unwind," and, unless there is some coherent source of these rolls elsewhere, the background state will tend to take over the spiral or target; on the other hand, if $q_{b}<q_{f}$ the target or spiral will tend to invade the background. Note that the invasive spirals rotate in the same direction as their chirality. The question of what to assume for $q_{b}$ is quite hard, and in the dynamic state a range of values is to be expected. We propose that since the background state often contains many stationary or slowly moving roll dislocations, a typical value for $q_{b}$ is the value $q_{d}$ of the wave number at which an inserted dislocation has zero velocity along the rolls (climb velocity) $[9,10]$. This leads to the prediction that spiral or target chaos is expected for roughly $q_{d}<q_{f}$.

The combined motion of a spiral and dislocation can be understood more fully by considering the situation shown in Fig. 1 with a single dislocation terminating a spiral at radius $R$. The motion can be split into two components. There is an outward motion of the rolls, driven by the curvature, at a speed $\alpha\left(q_{f}-q\right) / q R$ with $\alpha$ a positive proportionality constant and $q$ the wave vector at the dislocation: this would leave the spiral as shown by the dotted line. On the other hand, the dislocation will climb at a speed $\beta\left(q-q_{d}\right)$, moving in the azimuthal direction and shortening the spiral, with $\beta$ another positive proportionality constant. A steady state solution in which the spiral rotates at constant rate and with constant length exists if these effects cancel, i.e., if $q=\left(\alpha q_{f}+\beta q_{d}\right) /(\alpha+\beta)$, a weighted mean of $q_{f}$ and $q_{d}$.

We have investigated this picture in numerical simulations of equations modeling the convection system as in the work of $\mathrm{Xi}$, Gunton, and Vinals [3]. In this approach the system is described by an equation for a real field $\psi(\vec{x}, t)$, a function of horizontal coordinates and time only, that represents the horizontal structure of the convection pattern. The dynamical equation for $\psi$ is based on the Swift-Hohenberg equation [11] that has been much used in the study of stationary patterns and transient relaxation processes [7]:

$$
\begin{aligned}
\dot{\psi}+\vec{U} \cdot \vec{\nabla} \psi= & \epsilon \psi-\left(\nabla^{2}+1\right)^{2} \psi-g \psi^{3} \\
& +3(1-g)(\nabla \psi)^{2} \nabla^{2} \psi,
\end{aligned}
$$

where again $\vec{U}$ is the vorticity induced mean flow, driven by distortions of the field $\psi$. For $g=1 \mathrm{Eq}$. (5) reduces to the Swift-Hohenberg model. The additional nonlinear term [12] for $g \neq 1$ yields a more accurate reproduction of the fluid stability balloon and provides an additional tuning parameter to investigate transitions in behavior. We report here results for the same form of vorticity

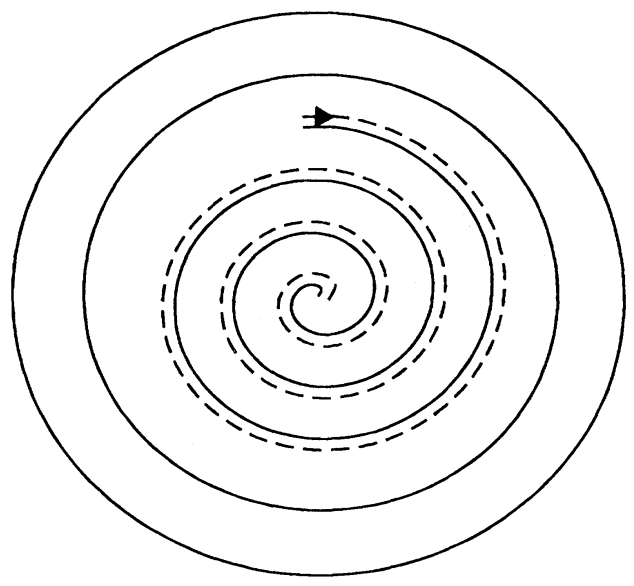

FIG. 1. Spiral defect ending in a dislocation. 
driving used by Xi, Gunton, and Vinals [3]:

$$
\dot{\Omega}-\sigma\left(\nabla^{2}-c^{2}\right) \Omega=g_{m} \hat{z} \cdot \vec{\nabla}\left(\nabla^{2} \psi\right) \times \vec{\nabla} \psi,
$$

where $\sigma$ is the fluid Prandtl number and $g_{m}$ determines the strength of the coupling between the mean flow and $\psi$. We have also studied the case of "filtered vorticity". introduced in Ref. [12], and "passive vorticity" with no $\dot{\Omega}$ term with similar results.

We have fixed $\epsilon=0.7, \sigma=1, c^{2}=2$, as in Ref. [3], and have varied the vorticity coupling $g_{m}$ and the nonlinearity coefficient $g$. Our simulations are done in a square geometry with periodic boundary conditions using the same numerical scheme as in Ref. [13]. A dynamic spiral or target state rapidly forms from random initial conditions over a wide range of parameters. We indeed see spirals rotating in either direction relative to their chirality, although corotating spirals are much more numerous, as expected.

We have studied the defect chaos state as a function of $g_{m}$ and $g$ to assess the importance of $q_{f}$ and $q_{d}$. Figure 2 shows the spatial structure at time 4000 after random initial conditions in a system of size $200 \times 200$ using dynamic, unfiltered vorticity. Panel (a) shows the dynamic spiral state for $g_{m}=50$ and $g=1$. As $g_{m}$ is reduced targets become more widespread, e.g., panel (b) with $g_{m}=10$. Finally, as $g_{m}$ is reduced further, panel (c) with $g_{m}=2$, the target-spiral state disappears, and the pattern freezes into a disordered stripe state. If alternatively $g$ is reduced, changing the form of the nonlinearity, the spatial structure remains rather unchanged, until at low $g$ again the spiral structures disappear, but now to a new dynamic state in which dislocation pairs, evident in panel (d) with $g=0.2$, rapidly migrate across the system. The quantitative aspects (a)

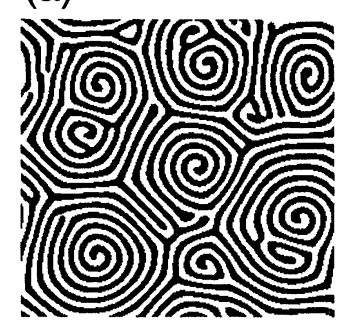

(c)

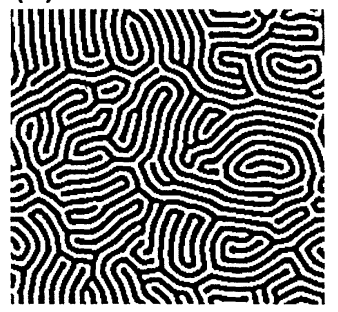

(b)

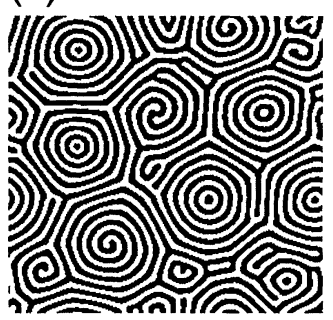

(d)

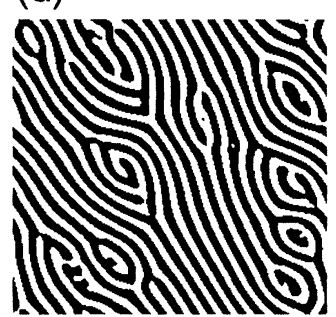

FIG. 2. Disordered states at time 4000 after random initial conditions in a system of size $200 \times 200$ for dynamic unfiltered vorticity showing the variation of the patterns as $g$ and $g_{m}$ are varied. Values are (a) $g=1, g_{m}=50$; (b) $g=1, g_{m}=10$; (c) $g=1, g_{m}=2$; (d) $g=0.2, g_{m}=50$. of the evolution of the structures with $g_{m}$ and $g$ are shown in Fig. 3. Both as a function of $g_{m}$ and $g$ the mean wave vector is seen to follow a trend consistent with a weighted mean of $q_{f}$ and $q_{d}$, except for the lowest $g_{m}$ where, in fact, the spatial structure shows few spirals or targets. In addition, the spiral or target state disappears when $q_{d}$ approaches $q_{f}$, although we do not have a model for which the $q_{f}$ and $q_{d}$ lines actually cross. It should also be noted that the wave vector distribution is not determined by the stability boundaries. This is shown, for example, in (b) where the distribution varies with $g_{m}$, whereas the Eckhaus stability boundary does not. The irrelevance of the crossroll instability is shown by similar results (not shown) for the model with filtered vorticity [12] where the cross-roll line is suppressed to larger wave vectors.

Further insight into the spiral-target transition is given by considering the dynamics of a single target in an axisymmetric geometry. Figure 4 shows time independent solutions constrained to axisymmetry in a circular geometry with boundary conditions $\psi=\partial \psi / \partial r=0$ that act to pin the phase of the rolls at the outer boundary. As the radius is increased, the rolls are stretched and the value of the field at the center goes up or down, until eventually a new roll is nucleated at the center. For the parameters used in Fig. 4 this event is hysteretic; if the radius is decreased
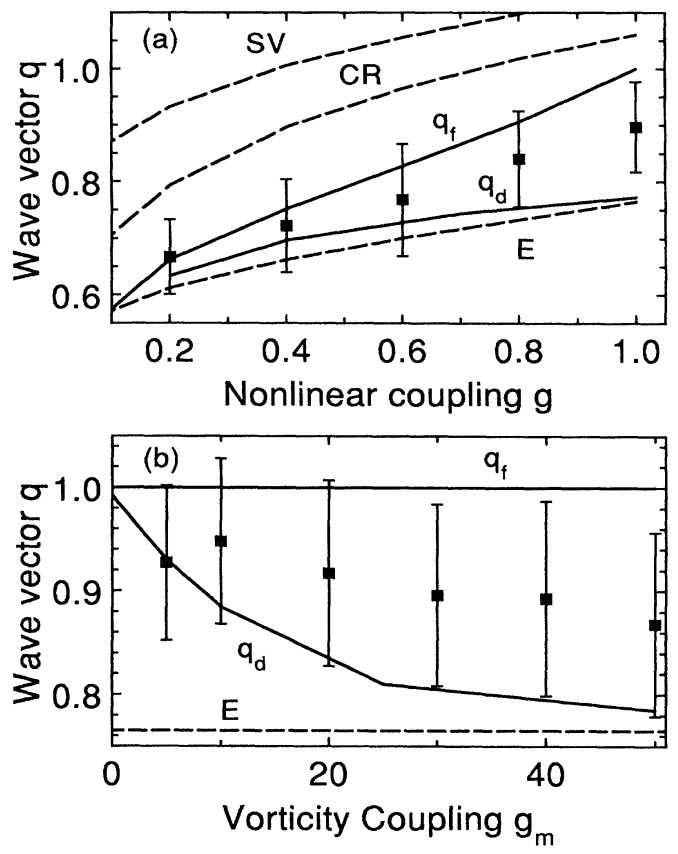

FIG. 3. Wave number distribution in disordered states as in Fig. 2 as a function of (a) $g$ for $g_{m}=50$, and (b) $g_{m}$ for $g=1$. The symbols are the mean wave vector and the error bars give the standard deviation, both given by a Gaussian fit to the distribution. The lines show $q_{f}$ and the wave vectors for the characteristic instabilities of straight parallel rolls (E: Eckhaus; SV: skew varicose; CR: cross roll) [14], and $q_{d}$ given by separate numerical calculations of the velocity of climb of a pair of dislocations in straight roll backgrounds of various wave vectors, as in Ref. [10]. 

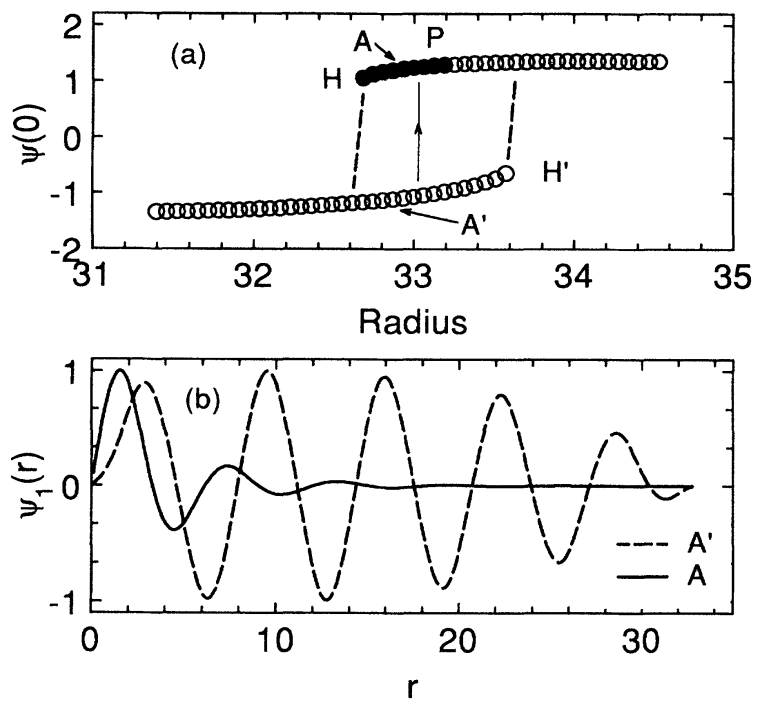

FIG. 4. (a) Variation of the field $\psi(r=0)$ at the center of a target as a function of the radius of the system. Notice the hysteretic transition. The states represented by the filled symbols are unstable to an $m=1$ distortion. The vertical arrow denotes a possible jump in a dynamic target state, leading to an $m=1$ unstable state. (b) Radial dependence of the eigenvector of the unstable mode at the point $A$ as well as the stable eigenvector with largest eigenvalue at the corresponding point $A^{\prime}$ on the stable branch. Notice the localized nature of the instability. Parameters are $g=1, g_{m}=50$, and $\epsilon=0.7$.

the roll disappears at a smaller radius than the one at which the nucleation occurred. Numerical linear stability analysis shows that the portion of the curve with filled symbols in Fig. 4 is unstable to an $m=1$ symmetry breaking perturbation (the most unstable mode). The eigenvector of the instability (Fig. 4) is localized at the core of the target, in contrast to the instability studied by Newell, Passot, and Souli [15]. As $g_{m}$ is decreased, the instability point $P$ moves closer to the point $H$ where the hysteretic jump occurs, and reaches this point at $g_{m} \simeq 21.5$ so that for smaller values of $g_{m}$ all states in the hysteresis loop are stable to $m=1$ perturbations.

In the dynamics of a target in a disordered background (e.g., Fig. 2) we can suppose that the target will approximately pass through the sequence of states represented in Fig. 4(a), with roll radiation corresponding to increasing the radius. We cannot say exactly where the jump from one branch to the other, corresponding to the nucleation of a new roll, will occur. However, if the nucleation occurs sufficiently early (e.g., as shown in the figure), the core will experience the nonaxisymmetric instability providing $g_{m}$ is greater than about 20 . This result is roughly consistent with the trend seen in our numerics Fig. 2 from mainly targets at $g_{m}=10$ to spirals at $g_{m}=50$.

In conclusion, we have proposed that the novel spiral or target chaos state may be understood in terms of the invasive dynamics of these defects. This dynamics in which rolls are radiated from the center is driven by wave vector frustration. For the spirals the rotation induced by the vertical vorticity plays a secondary role in the dynamics. The invasive dynamics depends on the balance between the focus selected wave vector $q_{f}$ and the background wave vector. The vorticity is, however, important in generating the spiral core, and the target to spiral transition may be partially understood in terms of a nonaxisymmetric instability of the axisymmetric core structure of a target. In addition, in the absence of the coupling to the vertical vorticity, the wave numbers less than $q_{f}$ that are expected in the far field are zigzag unstable [7], so that the vorticity is also important in stabilizing the pattern. Our proposal provides a criterion for when spiral chaos may be expected. The weakest part of our analysis is the identification of $q_{d}$ as a typical background wave vector: alternative possibilities might be a wave vector selected by another defect in the system that acts as a sink of the rolls, or a wave vector at which a roll destruction process develops, for example, by the production of dislocation pairs or the nucleation of a mobile dislocation from the core of a stationary defect. In addition, our analysis involves idealizations that may not be quantitatively accurate in all situations (e.g., we discuss well formed defects of large extent compared with the roll size). Nevertheless, we believe these ideas are important in understanding this novel and complex spatiotemporal state.

*Present address: IBM Thomas J. Watson Research Center, P.O. Box 218, Yorktown Heights, NY 10598.

[1] S. W. Morris, E. Bodenshatz, D. S. Cannell, and G. Ahlers, Phys. Rev. Lett. 71, 2026 (1993).

[2] M. Assenheimer and V. Steinberg, Phys. Rev. Lett. 70, 3888 (1993); Nature (London) 367, 345 (1994).

[3] H. Xi, J.D. Gunton, and J. Vinals, Phys. Rev. Lett. 71, 2030 (1993).

[4] W. Decker, W. Pesch, and A. Weber, Phys. Rev. Lett. 73, 648 (1994).

[5] M. C. Cross and A.C. Newell, Physica (Amsterdam) 10D, 299 (1984).

[6] A. C. Newell, T. Passot, and M. Souli, J. Fluid Mech. 220, 187 (1990).

[7] M. C. Cross and P.C. Hohenberg, Rev. Mod. Phys. 65, 851 (1993).

[8] Y. Pomeau and P. Manneville, J. Phys. (Paris) 42, 1067 (1981).

[9] Y. Pomeau, P. Manneville, and S. Zaleski, Phys. Rev. A 27, 2710 (1983).

[10] G. Tesauro and M.C. Cross, Phys. Rev. A 34, 1363 (1986).

[11] J. B. Swift and P. C. Hohenberg, Phys. Rev. A 15, 319 (1977).

[12] H. S. Greenside and M. C. Cross, Phys. Rev. A 31, 2492 (1985).

[13] M. C. Cross, Y. Tu, and D. I. Meiron, Chaos 4, 595 (1994).

[14] We thank H.S. Greenside and S. Wang for providing us with the code for performing the stability analysis.

[15] A. C. Newell, T. Passot, and M. Souli, Eur. J. Mec. B 1, 151 (1991). 